ISSN 0001-6002/2001/43/1/32-35

Acta Médica Costarricense,(C2001

Colegio de Médicos y Cirujanos

\title{
La Linfadenectomía Retroperitoneal para el Cáncer Testicular
}

\author{
Jaime Gutiérrez-Góngora, ${ }^{1}$ Claudio Orlich-Castelán ${ }^{1}$ y Arieh Grunhaus-Zelkowicz ${ }^{1}$
}

\begin{abstract}
Resumen: Históricamente, los pacientes diagnosticados con enfermedad neoplásica testicular B (II) han sido tratados con una disección primaria de ganglios retroperitoneales con la opción de aplicar quimioterapia adyuvante. Recientemente, varios autores han optado por la quimioterapia como tratamiento primario con la linfadenectomía retroperitoneal reservada para los pacientes que no lograban una remisión completa después del tratamiento con quimioterapia. El objetivo de este trabajo es definir por qué la linfadenectomía retroperitoneal (LR) es realizada siempre en el Servicio de Urología del Hospital San Juan de Dios en el tratamiento del cáncer testicular de células germinales no seminomatosas estadio clínico A (I) y B (II). Se presenta el sustento científico que avala esta práctica y se hace una breve reseña del estadiaje, del procedimiento quirúrgico utilizado.
\end{abstract}

Recibido: 5 de setiembre de 2000

Aceptado para publicación: 30 de enero de 2001

\section{Estadiaje}

La clasificación que se utiliza en el Servicio de Urologia del Hospital San Juan de Dios para los tumores testiculares de células germinales no seminomatosas es la de Boden y Gibb que establece el estadío A (I) como un tumor limitado al testículo y el B (II) aquel que tiene ganglios retroperitoneales positivos. Para propósito de este trabajo no se utiliza la modificación de Skinner del estadío B (define como BI aquel con menos de 6 ganglios positivos ninguno mayor de $2 \mathrm{~cm}$., B2 el de más de 6 ganglios positivos cualquiera mayor de $2 \mathrm{~cm}$., y el B3 el que tiene enfermedad retroperitoneal masiva).

El diagnóstico de estadío B (II) se basa en el estadiaje clínico con tomografía axial computada (TAC) del abdomen además del resultado de los marcadores tumorales principalmente alfa feto proteína. También se incluye una placa de torax en el estadiaje o un TAC del tórax si existen sospechas de metástasis en la primera. En el estadío B (II) se incluyen también aquellos pacientes sin evidencia radiológica de enfermedad retroperitoneal pero con elevación persistente de los marcadores tumorales.

Abreviaturas: col, colaboradores; LR, linfadenectomía retroperitoneal; TAC, Tomografía axial computada.

1. Servicio de Urología, Hospital San Juan de Dios.

Correspondencia: Jaime Gutiérrez Góngora. Apartado postal 99-2050 San Pedro. San José, Costa Rica.

\section{Procedimiento Quirúrgico}

Se utiliza el abordaje anterior al retroperitoneo (transabdominal, con una incisión mediana del xifoides casi a la sínfisis púbica) recomendado por Donohue y otros. ${ }^{1}$ En nuestra experiencia, esta técnica permite una visualización completa del espacio retroperitoneal pertinente para lograr una linfadenectomía efectiva. No se ha encontrado dificultad, excepto en casos de hombres con obesidad mórbida, con la disección en el área de los vasos renales y la zona suprahiliar, a menudo logrando identificar el orígen del tronco celíaco.

Los contenidos intestinales se manejan, en general, de dos formas. Una es la exposición del espacio retroperitoneal por medio de la retracción del colon descendente hacia la izquier$\mathrm{da}$, el colon transverso hacia arriba y el colon ascendente y todo el intestino delgado hacia la derecha. Si la cavidad peritoneal es pequeña o si el paciente es muy gordo se puede recurrir a la exteriorización del intestino delgado. Otra técnica que se utiliza en nuestro servicio es la movilización del intestino delgado y del colon ascendente por medio de una incisión del peritoneo posterior, que parte de la base del mesenterio del intestino delgado hacia el ciego y que se extiende por el aspecto lateral del colon derecho hacia arriba para luego colocar los contenidos intestinales en una bolsa plástica.

El patrón de la disección que se practica es el que recomendó Donohue y col. ${ }^{2}$ y en los hallazgos clínicos reportados por Narayan y col. ${ }^{3}$ que demostraron que una modificación de los bordes quirúrgicos permitía la eventual restauración de la eyaculación. Subsecuentemente, Richie estableció el méto- 
do que se utiliza en el Servicio de Urología del Hospital San Juan de Dios. La plantilla incluye todo el tejido retroperitoneal superior a los vasos renales y se extiende en ambos lados hasta el orígen del uretero respectivo. Del lado ipsilateral baja hasta el punto que el uretero cruza la arteria iliaca común y del lado contralateral hasta el orígen de la arteria inferior mesentérica. Con esta plantilla se preserva la eyaculación en más del $90 \%$ de los pacientes.

La mobilización extensa de los contenidos peritoneales siempre produce un ileo paralítico que se trata con un tubo nasogástrico y ambulación precoz y que infrecuentemente ha prolongado la estancia hospitalaria.

\section{Discusión}

La LR permite un estadiaje más preciso del retroperitoneo. "Aún con la tecnología del TAC de segunda generación y con el uso de los marcadores tumorales, el estadiaje clínico falsopositivo en nuestras manos sigue siendo significativamente inexacto (23\%)". 4 Esto significa que sin el beneficio de la L.R. el $23 \%$ de los pacientes son sometidos a la toxicidad que causa la quimioterapia a corto y largo plazo sin necesidad.

En el estadío clínico A, la LR permite la identificación inmediata de los ganglios positivos en el retroperitoneo. "En el estadío clínico A existe una tasa de falsos negativos del 30\%". ${ }^{4}$ Esto significa que cuando los pacientes con un estadío clínico A de bajo riesgo (sin invasión de vasos, de túnica albugínea o sin la presencia de cáncer embrionario) se observan, se está permitiendo que en el $30 \%$ de ellos el tumor aumente de volúmen mientras que con una LR se hubiera esterilizado el retroperitoneo inmediatamente, siendo confirmada esta tasa por otros investigadores. ${ }^{5}$

"La LR sola ofrece un beneficio terapeútico cuando los ganglios son positivos en dos terceras partes de los pacientes". ${ }^{4}$ El 63\% de los pacientes con enfermedad estadío B se curan sin los riesgos de la quimioterapia. En el otro tercio, la quimioterapia adyuvante resulta en una sobrevida del $98.3 \%$ en la enfermedad estadío B. 4 "Además, el $30 \%$ de los pacientes con estadío clínico B tratados con quimioterapia no logran una remisión completa mostrando una persistencia de enfermedad radiológicamente evidente en el retroperitoneo ${ }^{6}$ y requieren cirugía post-quimioterapia." 4 Esta tasa de fracaso es comparable a la que se obtiene con la LR.

La morbilidad de la LR es menor que la de la quimioterapia. "A largo plazo se logran remisiones completas después de unas pocas horas de cirugía y los resultados son comparables a la alternativa de 3 ciclos de quimioterapia combinada que duran 9 semanas o después de 4 ciclos que duran 12 semanas." 4

La LR es el procedimiento de escogencia en ciertos tipos de cánceres que no responden a la quimioterapia. Recientemente Leibovitch y col. reportan un $17 \%$ invasión ganglionar en el material de una linfadenectomía retroperitoneal en pacientes con teratoma maduro del testículo. Heindenreich y col. reportan que la frecuencia de mestástasis ganglionares fue del $19.2 \%$ en el estadío clínico 1 y del $66 \%$ en pacientes con estadío 2A/B. ${ }^{7}$ En general, el $43 \%$ de los pacientes con teratoma puro presentó enfermedad metastásica lo cual establece el potencial maligno del teratoma maduro testicular. ${ }^{8}$ Estos tumores no responden a la quimioterapia con Cisplatino como lo hacen los tumores de células germinales. ${ }^{8}$

La presencia de un teratoma en el tumor testicular, por lo tanto, prácticamente obliga a hacer una LR. Las metástasis ganglionares de alto volumen (estadíos II y III) y la presencia de elementos teratogénicos en el tumor primario son mejor tratados con una disección ganglionar retroperitoneal para evitar la doble terapia, puesto que la LR sola es suficiente para curarla, mientras que la quimioterapia primaria en el estadío II B logra solo una respuesta radiológica completa en el 33\% de los pacientes con teratoma en el tumor primario. ${ }^{9}$

Además, existe una alta incidencia de teratoma residual en el retroperitoneo en pacientes con teratoma en el tumor primario, que han sido tratados con quimioterapia primaria. ${ }^{9} \mathrm{~A}$ pesar de la apariencia benigna de los teratomas maduros que persisten después de la quimioterapia, éstos pueden tener un comportamiento maligno subsecuente ${ }^{10}$ que es otro buen argumento para efectuar una resección primaria de ganglios linfáticos retroperitoneales en este subgrupo de pacientes. ${ }^{9}$

La presencia en el tumor primario de carcinoma embrionario o invasión vascular también obliga a efectuar una LR. La combinación de un alto porcentaje de carcinoma embrionario y de invasión vascular predicen que habrá enfermedad retroperitoneal en el 92\% de esos pacientes con estadío clínico I. ${ }^{11}$

En los pacientes con niveles elevados de alfa fetoproteína antes de la orquiectomía, la incidencia de ganglios positivos es dos veces más elevada ${ }^{11}$ lo cual establece la utilidad de una LR primaria.

Ventajas de la LR sobre la quimioterapia:

a) Complicaciones de la LR comparadas con las de la quimioterapia. Las complicaciones de la LR fluctúan entre el 0 y el $10 \%$, mientras que se estima que la toxicidad a largo plazo de la quimioterapia fluctúa entre el 10 al $30 \% .{ }^{4}$ El papel de la Bleomicina en el tratamiento quimioterapeútico de los tumores testiculares de células espermáticas no seminomatosas es crucial, en el sentido de que hay un número mucho mayor de fracasos, cuando no se incluye la Bleomicina en el esquema quimioterapeútico. ${ }^{12}$ Sin embargo, la neumonitis inducida por Bleomicina es fatal en aproximadamente el 3\% de los pacientes tratados con este agente. ${ }^{13}$

b) La toxicidad de la quimioterapia ocurre en el $100 \%$ de los pacientes con una toxicidad gastrointestinal de moderada a severa en el 36\% de los pacientes durante la quimioterapia y parestesias periféricas o tínitus, en el $46 \%$ de ellos. ${ }^{14}$ 
c) Mortalidad de la LR comparada con la de la quimioterapia. La tasa de mortalidad es del $2 \%$ con la LR versus el $4 \%$ con el tratamiento de quimioterapia primaria. ${ }^{4}$

d) Las recidivas tardías (más de 2 años) después de la LR son del 0 al 1\%, mientras que con la quimioterapia primaria es del 3 al 5\%. ${ }^{4}$ En la experiencia de la Universidad de Indiana, con 464 pacientes con estadío clínico I sometidos a una disección de ganglios retroperitoneales de 1965 a 1989, sólo 3 tuvieron una recidiva tardía (0.6\%) y ésta ocurrió en el tórax. O sea, ninguno desarrolló una recidiva tardía en el retroperitoneo. Los autores concluyen que puesto que la recurrencia retroperitoneal es infrecuente después de una disección bien efectuada, una recidiva tardía en el retroperitoneo presumiblemente se relaciona con una técnica quirúrgica inadecuada. ${ }^{14}$

En el estudio de Baniel y col., el 58\% de los pacientes con recidivas tardías en el área retroperitoneal habían recibido quimioterapia adyuvante después de la disección de ganglios linfáticos retroperitoneales y, teóricamente, la quimioterapia debió haber evitado cualquier futura recurrencia. Los resultados de la quimioterapia para la recidiva tardía fue mala. Sólo 6 de los 35 pacientes que recibieron quimioterapia primaria para recidivas tardías tuvieron una respuesta completa y esta fue apenas transitoria. El único tratamiento exitoso en estos pacientes refractarios a la quimioterapia fue la resección quirúrgica del tumor localizado. ${ }^{15}$

Infertilidad después de la LR comparada con la vista postquimioterapia. La infertilidad a largo plazo después de la LR fluctúa entre el 20 al 30\% mientras que 6 meses después de tratamiento con quimioterapia basada en Cisplatino, Etoposida y Bleomicina todos los pacientes tienen azoospermia. A los dos años, el $74 \%$ tienen oligospermia y el $25 \%$ azoospermia. ${ }^{16}$

Se han reportado otras neoplasias primarias en pacientes después del tratamiento con quimioterapia primaria. ${ }^{17}$

Transformación del tumor original por la quimioterapia y mortalidad asociada: El $8.1 \%$ de los pacientes a quienes se les practicó una LR después de un tratamiento con quimioterapia tenían en los ganglios resecados elementos neoplásicos tipo sarcoma (con muerte enfermedad-específica del 31.6\%), cáncer no-sarcomatoso (con muerte enfermedad-específica del $45.4 \%$ ) y tipo coriocarcinoma quístico atípico (con muerte enfermedad-específica del $12.5 \%){ }^{3}$

El seguimiento después de la LR se facilita porque una evaluación periódica del espacio retroperitoneal por TAC es innecesaria después de la LR.

El modelo de la enfermedad es transformado después de la LR porque la recidiva en el retroperitoneo es infrecuente después de este procedimiento y cuando ocurre una recurrencia, esta se produce generalmente en los pulmones donde es mas fácil su detección. ${ }^{18}$
Los pacientes con tumores de células germinales no seminomatosas estadío clínico 1 que presentan marcadores tumorales séricos persistentemente elevados después de la orquiectomía se asume que tienen enfermedad metastásica oculta en ganglios linfáticos retroperitoneales. Los urólogos generalmente recomiendan una linfadenectomía retroperitoneal, agregando la quimioterapia adyuvante solo en aquellos pacientes con un estadío patológico $\mathrm{N} 2$ o N3 o en aquellos que tienen una elevación persistente de los marcadores tumorales después de la linfadenectomía. Davis y col. y Saxman y col. ${ }^{19}$ en estudios separados presentan evidencia de que estos pacientes con marcadores elevados también tienen un alto riesgo de recurrencia después de una disección de ganglios linfáticos retroperitoneales y establecen que estos pacientes deben ser tratados inicialmente con quimioterapia. ${ }^{10}$

\section{Conclusión}

Con base en la revisión de literatura urológica y a lo expuesto previamente, el Servicio de Urología del Hospital San Juan de Dios practica, y recomienda, la linfadenectomía retroperitoneal como procedimiento estándar en el tratamiento y manejo del cancer no seminomatoso testicular en los estadíos A y B (I y II).

\section{Abstract}

The purpose of this study is to report the rationale behind the use of retroperitoneal node dissection in patients with a non seminomatous germ cell carcinoma of the testis clinical stages $A(I)$ andB(II) in the Urology Department of the San Juan de Dios Hospital. We present the scientific basis for this practice and provide a short summary of the staging and surgical procedure.

Historically patients diagnosed with stage B(II) have been treated with primary dissection of the retroperitoneal lymph nodes with an option of adyuvant chemoterapy. Recently, various authors have chosen chemoterapy as the primary treatment while retroperitoneal lymphadenectomy is reserved for patients who do not achieve a complete remission after chemoterapy.

The Department of Urology at the San Juan de Dios Hospital routinely performs a retroperitoneal lymph node dissection for patients with a non seminomatous germ cell carcinoma of the testis stages A and B (I and II).

\section{Referencias}

1. Rowland RG, Donohue JP. El cáncer testicular: novedades en el diagnóstico y tratamiento. Semin Urol 1988; 6 (3): 223-232.

2. Donohue JP, Zachary JM, Maynard BR. La distribución de las metástasis ganglionares en el cáncer testicular no seminomatoso. J Urol 1982, 128 (2): 315-320.

3. Narayan P, Lange PH, Fraley EE. La eyaculación y la fertilidad después de una disección de ganglios linfáticos retroperitoneales extensa para el cáncer testicular. J Urol 1982; 127 (4): 685-8. 
4. Donohue JP, Thornhill JA, Foster RS, Rowland RG, Bihrle R. El papel de la linfadenectomía retroperitoneal en el cáncer testicular estadío B: la experiencia de la Universidad de Indiana (1965-1989). Eur J Cancer 1995; 31 A (10). 1599-604.

5. de Riese WT, Albers P, Walker EB, Ulbright TM, Crabtree WN, Reister T, Foster RS, Donohue JP. Parámetros que predicen el comportamiento biológico del estadío temprano de los tumores de células germinales no seminomatosas. Cáncer 1994; 74 (4): 1335-41.

6. Littler JS Jr, Foster RS, Ulbright TM, Donohue JP. Neoplasias inusuales detectadas en pacientes con cáncer testicular sometidos a una linfadenectomía retroperitoneal después de la quimioterapia. J Urol 1994; 152(4): 1144-9.

7. Heidenreich A, Moul JW, McLeod DG, Mostofi FK, Engelmann UH. El papel de una linfadenectomía retroperitoneal en el teratoma maduro del testículo. J Urol 1997; 157 (1): 160-3.

8. Motzer RJ, Amsterdam A, Prieto V, Sheinfeld J, Murty VV, Mazumdar $\mathrm{M}$, et. al. El teratoma con transformación maligna: diferentes histologías malignas surgiendo en hombres con tumores de células germinales. J Urol 1998; 159 (1): 133-8.

9. Rabbani F, Gleave ME, Coppin CM, Murray N, Sullivan LD. Un teratoma en el tumor testicular primario reduce la tasa de una respuesta completa en el retroperitoneo después de la quimioterapia primaria: la indicación para una disección primaria de ganglios linfáticos retroperitoneales de los tumores de células germinales estadío IIb con elementos teratomatosos. Cancer 1996; 78 (3): 480-6.

10. Nativ O, Shajrawi I, Leibovitch I, Moskovitz B. El potencial maligno del teratoma maduro residual después de la quimioterapia para tumores testiculares no seminomatosos diseminados. Eur Urol 1994; 26 (3): 216-8.

11. Moul JW, Heidenreich A. Los factores de pronóstico en el cáncer testicular no seminomatoso de bajo estadío. Oncology 1996; 10 (9): 1359-68.
12. Lehrer PJ, et al. La importancia de la Bleomicina en el pronóstico favorable de los tumores diseminados de células espermáticas. J Urol 1995; 154: 2206

13. Sleijfer S, van der Mark TW, Schraffordt Koops H, Mulder NH. Una disminución de la función pulmonar durante la quimioterapia combinada con Bleomicina para el cáncer testicular. Br J Cancer 1995; 71 (1): $120-3$.

14. Lerner SE, Mann BS, Blute ML, Richardson RL, Zincke H. La quimioterapia primaria para los tumores testiculares de células germinales no seminomatosas estadío clínico II: criterios de selección y resultados a largo plazo. Mayo Clin Proc 1995; 70 (9): 821-8.

15. Baniel J, Foster RS, Einhorn LH, Donohue JP. La recidiva tardía del cáncer testicular estadío clínico I. J Urol 1995; 154 (4): 1370-2.

16. Stephenson WT, Poirier SM, Rubin L, Einhorn LH. Evaluación de la capacidad reproductiva en pacientes con tumores de células germinales después del tratamiento con Cisplatino, Etoposida y Bleomicina. J Clin Oncol 1995; 13 (9): 2278-80.

17. Studer UE, Fey MF, Calderoni A, Kraft R, Mazzucchelli L, Sonntag RW. La quimioterapia adyuvante después de la orquiectomía en pacientes de alto riesgo con cáncer testicular no seminomatoso estadío clínico I. Eur Urol 1993; 23 (4): 444-9.

18. Birch R, Williams S, Cone A, Einhorn L, Roark P, Turner S, Greco FA. Factores de pronóstico para un resultado favorable en tumores diseminados de células germinales. J Clin Oncol 1986; 4 (3): 400-7.

19. Saxman SB, Nichols CR, Foster RS, Messemer JE, Donohue JP, Einhorn LH. El manejo de pacientes con tumores testiculares no-seminomatosos estadío clínico I con marcadores serológicos persistentemente elevados. J Urol 1996; 155 (2): 587-9. 